\title{
Wet Heat Exposure: A Potentially Reversible Cause of Low Semen Quality in Infertile Men
}

\author{
Shai Shefi, Phiroz E. Tarapore, Thomas J. Walsh, Mary Croughan, Paul J. Turek \\ Departments of Urology (SS, PET, TJW, PJT), Obstetrics, Gynecology and Reproductive Science \\ (MC, PJT), and Epidemiology and Biostatistics (MC), University of California San Francisco, San \\ Francisco, California, USA
}

\begin{abstract}
Objective: To evaluate the recovery of semen quality in a cohort of infertile men after known hyperthermic exposure to hot tubs, hot baths or whirlpool baths.

Materials and Methods: A consecutive cohort of infertile men had a history remarkable for wet heat exposure in the forms of hot tubs, Jacuzzi or hot baths. Clinical characteristics and exposure parameters were assessed before exposure was discontinued, and semen parameters analyzed before and after discontinuation of hyperthermic exposure. A significant seminal response to withdrawal of hyperthermia was defined as $\geq 200 \%$ increase in the total motile sperm count $(\mathrm{TMC}=$ volume $\mathrm{x}$ concentration $\mathrm{x}$ motile fraction) during follow-up after cessation of wet heat exposure.

Results: Eleven infertile men (mean age 36.5 years, range 31-44) exposed to hyperthermia were evaluated pre and postexposure. Five patients $(45 \%)$ responded favorably to cessation of heat exposure and had a mean increase in total motile sperm counts of $491 \%$. This increase was largely the result of a statistically significant increase in sperm motility from a mean of $12 \%$ at baseline to $34 \%$ post-intervention $(\mathrm{p}=0.02)$. Among non-responders, a smoking history revealed a mean of 5.6 pack-years, compared to 0.11 pack-years among responders. The prevalence of varicoceles was similar in both cohorts.

Conclusions: The toxic effect of hyperthermia on semen quality may be reversible in some infertile men. We observed that the seminal response to exposure elimination varies biologically among individuals and can be profound in magnitude. Among non-responders, other risk factors that could explain a lack of response to elimination of hyperthermia should be considered.
\end{abstract}

Key words: male infertility; induced hyperthermia; semen; analysis; spermatogenesis

Int Braz J Urol. 2007; 33: 50-7

\section{INTRODUCTION}

Spermatogenesis is sensitive to a variety of chemical and physical stressors. Testicular hyperthermia has been known to have a deleterious effect on male fertility since the time of Hippocrates and is a well-recognized cause of impaired sperm production
(1). Its detrimental effect has been demonstrated in both animal models and in humans (2-4). Whether due to endogenous (such as high fevers) or exogenous stimuli, heat decreases sperm concentration, impairs motility, and reduces the number of morphologically normal sperm (5-8). This effect is striking enough that the effect of laptop computers on scrotal hyperther- 
mia has recently been reported and the use of heat exposure as a male contraceptive has been studied $(9,10)$. In contrast to the well described detrimental effects of dry heat on spermatogenesis, the consequences of wet heat exposure are relatively undefined. Our working hypothesis is that the effects of wet heat on spermatogenesis are similar to that from dry heat.

The only published study to examine the effects of wet heat exposure on human fertility was performed by Rock and Robinson in 1965. In this study, the authors exposed 20 oligospermic men to wet heat $\left(43-45^{\circ} \mathrm{C}\right)$ with a bottle warmer held between the thighs for 30 minutes on 6 alternating days. They noted a decline in sperm production that was followed by improvement in seminal parameters with cessation of the exposure, however the details of semen quality before and after exposure were not provided by the authors (11). The effects of more common exposures to wet heat, such as with hot tubs, Jacuzzis, or hot baths have not been addressed in the literature.

The data provided by Rock and Robinson, as well as the well-defined association between dry heat exposure and impaired spermatogenesis led us to study the effect of total-body wet heat exposure in human males. We asked whether withdrawal of exposure in men with poor semen quality and a history of wet heat exposure could lead to an improvement in semen quality. We retrospectively compared semen quality before and after cessation of wet heat exposure, and also performed an interval analysis of responders and non-responders, looking for factors that might modulate responsiveness to therapeutic intervention.

\section{MATERIALS AND METHODS}

Over a 3-year period, infertile men with exposure to wet heat were identified in a single, university-based, male infertility practice. Inclusion criteria were wet heat exposure, defined as the immersion of the body in a hot tub, heated Jacuzzi, or bath at a temperature warmer than body temperature, for $\geq 30$ minutes per week during $\geq 3$ months prior to presentation. All female partners were concurrently assessed for reproductive issues. Patients were excluded if they had received any medical or surgical infertility treatment within 1 year of study intake, and if a diagnosis of co-existing female factor infertility was made. Because patient data was decoded of all protected health information, a waiver was obtained from the Committee on Human Research for this study.

All subjects underwent a complete history and physical examination by a single specialist (PJT). Information regarding the type, frequency, duration/ episode and overall length of exposure to wet heat was collected during this visit. Counseling regarding the cessation of wet heat exposure was given during the initial visit. We attempted to obtain 2 semen samples for analysis at baseline (during exposure), < 3 months after intervention, and between 3-6 months after intervention in all patients. Semen was collected by masturbation after 2 to 3 days of abstinence and processed within 1 hour of ejaculation. All semen analyses were performed in a single andrology laboratory by the same technician, and assessed according to World Health Organization guidelines (12). Motility was reported as the sum of $\mathrm{A}+\mathrm{B}$ patterns (12). A positive response to discontinuation of heat exposure was defined as an increase in total motile count $(\mathrm{TMC}=$ ejaculate volume $\mathrm{x}$ sperm concentration $\mathrm{x}$ fraction of motile sperm) $>200 \%$ during follow-up. This cutoff was selected to be above the natural variability in semen parameters within an individual: sperm volume, concentration and motility have been shown to vary by $59 \%, 54 \%$ and $96 \%$, respectively, in a single individual with time (13). Statistical analysis was performed using two-tailed, paired " $\mathrm{t}$ " test to compare pre- and post-intervention seminal parameters. Probability values $<0.05$ were considered significant.

\section{RESULTS}

\section{Patient Characteristics}

Table- 1 outlines the demographic and clinical characteristics of the 11 eligible study subjects. Mean patient age was 36.5 years, and mean partner age was 34.6 years. Couples had been trying to conceive for an average of 2.6 years (range 0 - 15 years) prior to evaluation. Nine patients $(82 \%)$ had an evi- 
Table 1 - Characteristics of the study group.

\begin{tabular}{lcc}
\hline Characteristic & Mean \pm SD & Range \\
\hline Patient age (yr) & $36.5 \pm 4.5$ & $31-44$ \\
Partner age (yr) & $34.6 \pm 6.4$ & $22-42$ \\
Time attempting (yr) & $2.6 \pm 4.3$ & $0-15$ \\
Exposure (min/week) & $148.6 \pm 82.3$ & $60-315$ \\
Exposure history (yr) & $2.9 \pm 3.3$ & $0.75-10$ \\
R-testis volume (mL) & $16.9 \pm 3.7$ & $10-20$ \\
L-testis volume (mL) & $17.0 \pm 4.9$ & $8-25$ \\
Smoking (pack-year) & $3.1 \pm 5.5$ & $0-15$ \\
\hline
\end{tabular}

Testicular volume was determined using Prader orchidometer.

dence of infertility, defined as failure to conceive after one year of unprotected sexual intercourse. The mean exposure duration was 149 minutes per week (range 60 - 315), with a mean total duration of hyperthermic exposure of 2.9 years (range 0.75 - 10). Five subjects were exposed to hot baths, 4 to both hot baths and hot tubs, and 2 to Jacuzzi. Three of the 11 subjects had a significant smoking history ( $>6$ pack-years). Five others were only occasional smokers ( $<1$ pack-years). Complete pre-intervention semen parameters among study subjects are given in Table-2. Patients had an average of $1.6 \pm 0.6$ and $2.5 \pm 1.7$ semen analyses performed prior to cessation of exposure and following cessation, respectively. Nine men $(82 \%)$ had TMC <
Table 2 - Seminal parameters before intervention.

\begin{tabular}{lcc}
\hline Characteristic & Mean \pm SD & Range \\
\hline Ejaculate volume $(\mathrm{mL})$ & $3.3 \pm 1.3$ & $1.3-5.3$ \\
Sperm concentration $\left(\mathrm{x} 10^{6} / \mathrm{mL}\right)$ & $15.8 \pm 16.0$ & $0.2-42.5$ \\
Sperm motility $(\%)$ & $23.8 \pm 20.6$ & $0-70$ \\
Total motile sperm count $\left(\mathrm{x} 10^{6}\right)$ & $17.9 \pm 34.5$ & $0-118$ \\
\hline
\end{tabular}

$20 \times 10^{6}$ (mean $5 \times 10^{6}$ ). The other 2 men had TMC's of $34 \times 10^{6}$ and $117.6 \times 10^{6}$ sperm.

\section{Response to Cessation of Wet Heat Exposure}

Semen analyses for each subject were categorized into baseline (during exposure), 0 to 3 months post-intervention, and $>3$ months postintervention. Multiple semen analyses within a given period were averaged for each individual during that period. Sperm parameters before and after discontinuation of exposure are listed in Table-3. TMC values and response to intervention status are shown in Table-4. Mean follow-up of the study cohort was 7 months (range 2-16). Amongst the entire cohort, improvement in TMC did not reach statistical significance $(\mathrm{p}=0.89)$. Overall, $44.5 \%(95 \%$ CI $16.7,76.7)$ of men showed $>200 \%$ increase in total motile sperm count after discontinuation of wet heat

Table 3 - Semen parameters at baseline, 0-3 months post-intervention, and more than 3 months post-intervention.

\begin{tabular}{|c|c|c|c|c|c|c|c|c|c|}
\hline \multirow[b]{2}{*}{$\begin{array}{l}\text { Pt. } \\
\text { No }\end{array}$} & \multicolumn{3}{|c|}{ Baseline } & \multicolumn{3}{|c|}{ 0-3 months } & \multicolumn{3}{|c|}{ 3+ months } \\
\hline & $\begin{array}{c}\text { Volume } \\
(\mathrm{mL})\end{array}$ & $\begin{array}{c}\text { Concentration } \\
\left(\times 10^{6} / \mathrm{mL}\right)\end{array}$ & $\begin{array}{c}\text { Motility } \\
(\%)\end{array}$ & $\begin{array}{c}\text { Volume } \\
(\mathrm{mL})\end{array}$ & $\begin{array}{c}\text { Concentration } \\
\left(\times 10^{6} / \mathrm{mL}\right)\end{array}$ & $\begin{array}{c}\text { Motility } \\
(\%)\end{array}$ & $\begin{array}{c}\text { Volume } \\
(\mathrm{mL})\end{array}$ & $\begin{array}{c}\text { Concentration } \\
\left(\times 10^{6} / \mathrm{mL}\right)\end{array}$ & $\begin{array}{c}\text { Motility } \\
(\%)\end{array}$ \\
\hline 1 & 3.0 & 3.0 & 34 & 2.4 & 14.9 & 24 & 1.8 & 12.0 & 44 \\
\hline 2 & 5.3 & 10.5 & 17 & 3.5 & 18.0 & 62 & - & - & - \\
\hline 3 & 4.4 & 0.7 & 23 & 4.5 & 0.7 & 15 & 1.8 & 1.5 & 7 \\
\hline 4 & 4.0 & 42.0 & 70 & 2.5 & 63.0 & 55 & - & - & - \\
\hline 5 & 1.7 & 0.2 & 0 & 0.8 & 1.0 & 23 & - & - & - \\
\hline 6 & 4.2 & 9.5 & 37 & 6.0 & 7.0 & 23 & 4.6 & 9.0 & 32 \\
\hline 7 & 2.0 & 42.5 & 40 & - & - & - & 1.0 & 21.0 & 40 \\
\hline 8 & 2.5 & 13.0 & 9 & 3.5 & 21.0 & 3 & - & - & - \\
\hline 9 & 4.8 & 9.0 & 24 & 4.5 & 5.0 & 26 & 5.5 & 5.4 & 21 \\
\hline 10 & 3.4 & 8.9 & 2 & 3.3 & 15.0 & 36 & 3.6 & 19.0 & 19 \\
\hline 11 & 1.3 & 35.0 & 7 & 1.3 & 14.0 & 4 & 2.1 & 32.4 & 22 \\
\hline
\end{tabular}


Table 4-TMC $\left(\times 10^{6}\right)$ at baseline, 0-3 months post-intervention, and $>3$ months post-intervention.

\begin{tabular}{lrcccc}
\hline Pt. No. & Baseline & $\mathbf{0 - 3}$ months & 3+ months & Change (\%) & Responder \\
\hline 1 & 2.9 & 10.1 & 9.3 & +221 & Yes \\
2 & 9.5 & 39.1 & - & +312 & Yes \\
3 & 0.6 & 0.7 & 0.2 & -67 & No \\
4 & 117.6 & 86.6 & - & -26 & No \\
5 & 0 & 0.2 & - & $+\infty$ & Yes \\
6 & 14.4 & 9.7 & 13.2 & -8 & No \\
7 & 34 & 1.8 & 8.4 & -72 & No \\
8 & 2.9 & 5.9 & 5.5 & -47 & No \\
9 & 10.4 & 17.8 & 10.7 & +873 & Yes \\
10 & 1.1 & 0.7 & 21.1 & +559 & Yes \\
11 & 3.2 & & & - & -38 \\
\hline
\end{tabular}

$T M C=$ total motile sperm count, \% change: based on last available data compared to baseline for each individual. Response was defined as an increase of $T M C \geq 200 \%$.

exposure and were considered responders to intervention.

Among responders, the mean increase in TMC was $491 \%$ (range 221-873), excluding an outlier whose TMC improved from zero to 200,000 sperm. Responders' TMC before and after intervention are illustrated in Figure-1. The seminal response in the responding cohort showed increases in sperm concentration, but largely the result of increases in sperm motility. Although the increase in TMC increase did not reach statistical significance $(\mathrm{p}=$ 0.07 ) among responders, a subset analysis showed a mean increase of $22 \%$ in sperm motility, which was statistically significant $(\mathrm{p}=0.02)$. Among responders, one patient had a TMC increase from 0 to 0.2 million sperm, two patients had post-intervention TMC's of 9.3 and $10.7 \times 10^{6}$, while two more had a TMC's of $>$ $20 \times 10^{6}$ sperm. There was no significant difference in the length of follow-up among study responders and non-responders.

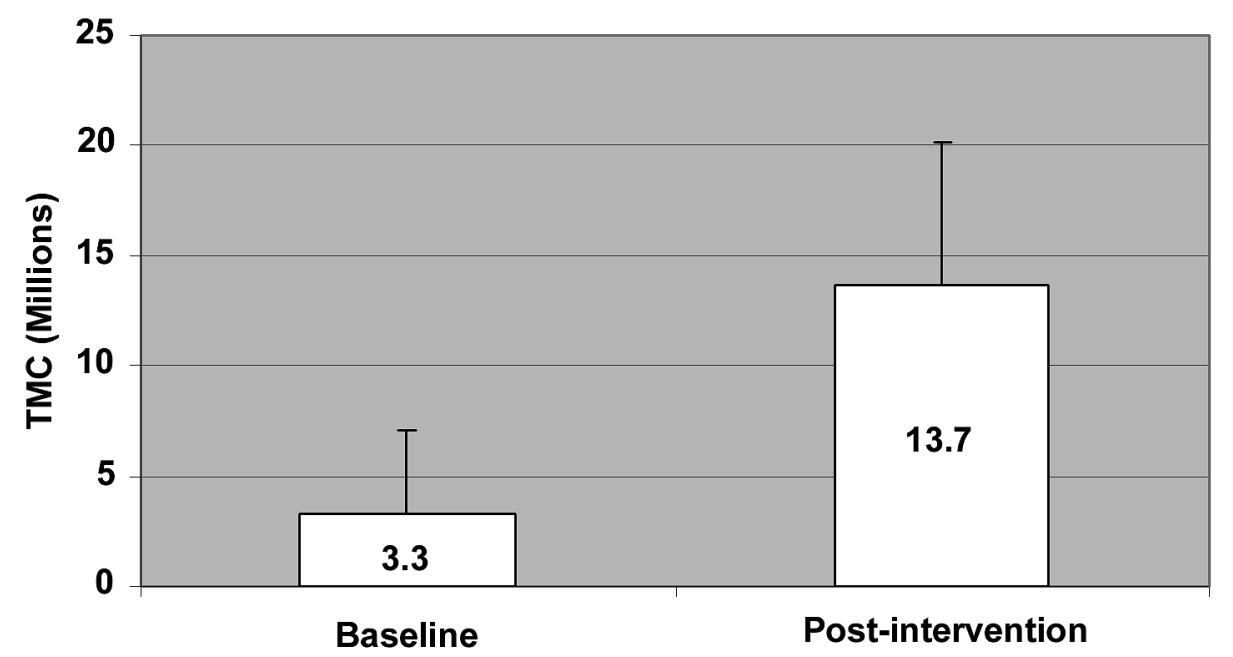

Figure 1 - Mean total motile count (TMC) in responders cohort for baseline and > 3 months post-intervention. Bars represent standard deviation. 
Factors that could explain a response or lack of response to the intervention were examined in both cohorts. Tobacco use emerged as a possible differentiating factor. Among 6 non-responders, 5 were chronic tobacco users with a significant smoking history (mean 5.6 pack-years), compared with 3 occasional smokers in the responder group, whose mean tobacco consumption was 0.11 pack-years. The prevalence of varicoceles was similar in responders and non-responders (4/5 and 4/6 patients, respectively). No other potential gonadotoxic factors were identified in the study cohort.

\section{COMMENTS}

The removal of wet heat exposure resulted in improvement in semen quality in nearly one-half of subjects studied. The improvement was largely the results of increases in sperm motility, and appeared to persist beyond 3 months, although a continuous improvement was not observed in all subjects. The extra-abdominal position of the scrotum and the proximity of the pampiniform venous plexus to the testicular arteries contribute to the efficient dissipation and transfer of heat away from the testes and resultant lower testicular temperature (8). However, in the presence of extreme elevation of extra-testicular temperature, as when immersed in hot liquid, these same characteristics make the scrotum particularly susceptible to deleterious thermal effect. It is therefore not surprising that semen quality might be susceptible to the effects of wet hyperthermia.

The deleterious effect of dry heat on semen quality and, by extrapolation, male fertility, has been recognized medically for decades, and to the traditional medicine community for millennia. Carlsen et al., in examining the effect of febrile illness on semen quality, demonstrated a dose-response relationship between the number of days with fever and sperm concentration (8). Similarly, Mieusset \& Bujan examined mild testicular heating (about $1^{\circ} \mathrm{C}$ ) as a form of contraception, an idea originally conceived of by Robinson et al. $(14,15)$. Both studies found that sperm concentration rebounded to baseline in 12 to 18 months following cessation of increased testicular temperatures. None of these studies, however, were designed to replicate the frequent, significant wet hyperthermia that is the fate of the habitual hot-tuber, thus forming the rationale for this study.

In reviewing our data on the recovery of semen quality in infertile males following the cessation of wet heat exposure, we found that there were two distinct groups of patients: those that responded to intervention and those that did not. Among responders, improvements in semen quality were witnessed well beyond the 3 month period typically ascribed to a single cycle of spermatogenesis. This finding is entirely consistent with the time course of recovery noted after varicocele repair and exposure to other gonadotoxins (16). Interestingly, the semen analysis parameter that exhibited the largest change among responders was sperm motility, and this increase reached statistical significance after intervention. This suggests that heat-induced motility dysfunction may be the parameter most vulnerable to wet hyperthermic exposure. Although statistical significance was not achieved in the overall increase in TMC after intervention, the changes observed could be considered clinically significant, as more men could qualify for IUI instead of IVF for infertility treatment following intervention. Given that intrauterine insemination (IUI) can be considered for low semen quality in men with TMC > 8-10 x $10^{6}$ sperm, the number of men who would qualify for IUI in the responder group increased from $1 / 5$ preintervention to $4 / 5$ post-intervention. Two of the responders had TMC $>20 \times 10^{6}$ after intervention and were advised to consider unprotected intercourse before IUI. This "shift of care" to less intensive forms of assisted reproduction has also been described for varicocele repair (16). We acknowledge that even statistically significant changes in semen parameters are always problematic to assess, given the naturally high intra-individual variability of semen parameters in both fertile and infertile men. However, the potential clinical value of these changes must also be considered, as we have outlined.

Careful analysis of non-responders suggests that chronic tobacco use was more common among non-responders, suggesting that it may complicate the recovery from wet heat exposure. This finding is hardly surprising, considering that tobacco use is a 
well established, independent risk factor for poor semen quality (17-19). It is interesting to speculate whether the concomitant exposure to tobacco and wet heat may act synergistically to prevent early and rapid recovery of semen quality.

It is interesting that two responders demonstrated subtle declines in TMC following the 3 -month time interval. This lack of continuous improvement suggests that peak improvements in TMC may be time-limited and could involve other, less well-described physiological responses. It may also suggest that a timeline for therapy be offered to affected patients, to avoid prolonged follow-up without obvious benefit.

Because this is a small study, the results should be confirmed in a larger series of patients. Given the complexity of factors underlying male infertility, including currently uncharacterized factors, more accurate results will only be generalizable with a larger study. We plan further follow-up on the patients in this study to assess conception after elimination of hyperthermia.

\section{CONCLUSIONS}

This study addressed the relatively unsubstantiated issue of wet heat exposure as a factor in male infertility. We demonstrated that infertile men who are frequently exposed to wet heat in the form of hot tubs, Jacuzzis, or hot baths, may realize a marked increase in semen quality following cessation of exposure. The response persists for more than 3 months, and is driven mostly by the increase in sperm motility.

\section{CONFLICT OF INTEREST}

None declared.

\section{ACKNOWLEDGEMENT}

Doctor Shai Shefi is Recipient of fellowship from The American Physicians Fellowship for Medicine in Israel

\section{REFERENCES}

1. Dada R, Gupta NP, Kucheria K: Spermatogenic arrest in men with testicular hyperthermia. Teratog Carcinog Mutagen. 2003; Suppl 1: 235-43.

2. Lue YH, Lasley BL, Laughlin LS, Swerdloff RS, Hikim AP, Leung A, et al.: Mild testicular hyperthermia induces profound transitional spermatogenic suppression through increased germ cell apoptosis in adult cynomolgus monkeys (Macaca fascicularis). J Androl. 2002; 23: 799-805.

3. Rockett JC, Mapp FL, Garges JB, Luft JC, Mori C, Dix DJ: Effects of hyperthermia on spermatogenesis, apoptosis, gene expression, and fertility in adult male mice. Biol Reprod. 2001; 65: 229-39.

4. Adams F (translator):The Genuine Works of Hippocrates. Baltimore, Wilkins \& Wilkins. 1939.

5. MacLeod J, Hotchkiss RS: The effect of hyperpyrexia upon. spermatozoa counts in men. Endocrinology. 1941; 28: 780-784.

6. Procope BJ: Effect of repeated increase of body temperature on human sperm cells. Int J Fertil. 1965; 10: 333-9.

7. Saikhun J, Kitiyanant Y, Vanadurongwan V, Pavasuthipaisit K: Effects of sauna on sperm movement characteristics of normal men measured by computerassisted sperm analysis. Int J Androl. 1998; 21: 358-63.

8. Carlsen E, Andersson AM, Petersen JH, Skakkebaek NE: History of febrile illness and variation in semen quality. Hum Reprod. 2003; 18: 2089-92.

9. Sheynkin Y, Jung M, Yoo P, Schulsinger D, Komaroff E: Increase in scrotal temperature in laptop computer users. Hum Reprod. 2005; 20: 452-5.

10. Kandeel FR, Swerdloff RS: Role of temperature in regulation of spermatogenesis and the use of heating as a method for contraception. Fertil Steril. 1988; 49: $1-23$.

11. Rock J, Robinson D: Effect of induced intrascrotal hyperthermia on testicular function in man. Am J Obstet Gynecol. 1965; 93: 793-801.

12. WHO Laboratory Manual for the Examination of the Human Semen and Sperm-Cervical Mucus Interaction. Fourth edition. Cambridge, Cambridge University Press. 1999.

13. Mallidis C, Howard EJ, Baker HW: Variation of semen quality in normal men. Int J Androl. 1991; 14: 99-107.

14. Mieusset R, Bujan L: The potential of mild testicular heating as a safe, effective and reversible contraceptive method for men. Int J Androl. 1994; 17: 186-91.

15. Robinson D, Rock J, Menkin MF: Control of human 
spermatogenesis by induced changes of intrascrotal temperature. JAMA. 1968; 204: 290-7.

16. Cayan S, Erdemir F, Ozbey I, Turek PJ, Kadioglu A, Tellaloglu S: Can varicocelectomy significantly change the way couples use assisted reproductive technologies? J Urol. 2002; 167: 1749-52.

17. Hull MG, North K, Taylor H, Farrow A, Ford WC: Delayed conception and active and passive smoking.
The Avon Longitudinal Study of Pregnancy and Childhood Study Team. Fertil Steril. 2000; 74: 72533.

18. Vine MF: Smoking and male reproduction: a review. Int J Androl. 1996; 19: 323-37.

19. Hughes EG, Brennan BG: Does cigarette smoking impair natural or assisted fecundity? Fertil Steril. 1996; 66: 679-89.
Accepted after revision:

October 18, 2006

\section{Correspondence address:}

Dr. Paul J Turek

Department of Urology, UCSF

1600 Divisidero St, Rm A633

San Francisco, CA, 94143-1695, USA

E-mail: pturek@urology.ucsf.edu

\section{EDITORIAL COMMENT}

Numerous factors can elevate scrotal temperature either by whole body or local scrotal heating. Scrotal hyperthermia was found in men with febrile illness, retractile testes, occupations associated with high temperature exposure, hot bath and sauna users, men wearing tight jockey shorts, car drivers, and laptop computer users. Multiple human studies have confirmed deleterious effect of increased scrotal temperature on sperm quantity and quality.

The authors investigate a less defined effect of total body wet heat exposure (hot tub, heated Jacuzzi or bath) on spermatogenesis. While studied population is very small and most results are not statistically significant, this article presents evidence of occasional reversibility of negative thermal effect and improvement of total motile sperm count and motility after cessation of heat exposure within the predictable interval of 3 months. However, time to maximum effect and durability of improvement also remain unclear due to a short follow-up period.

Despite significant limitations the study is noteworthy since it emphasizes important but frequently omitted details in the evaluation and management of the infertile men: 1) It is necessary to ask patients about habitual and occupational heat exposure, 2) It is worthwhile to recommend cessation of confirmed frequent heat exposure and repeat semen analysis within 3 month interval. Improved sperm quantity and quality in select patients may "upgrade" their treatment from IVF to IUI or, possibly, from IUI to natural pregnancy.

Finally, since such improvement, its magnitude and durability are not predictable, watchful waiting approach has to be exercise within strictly defined and limited time which will not delay other recommended treatment options.

Dr. Yefim R. Sheynkin Associate Professor of Clinical Urology State University of New York at Stony Brook

Stony Brook, New York, USA E-mail: ysheynkin@notes.cc.sunysb.edu 


\section{REPLY BY AUTHORS}

Although talked about for decades, the detrimental effect of hot tubs, Jacuzzis and hot baths on semen quality and male fertility has never been formally investigated, and forms the rationale for this study. As a result, we now have actual evidence to show patients that these recreational activities are a real risk factor for male subfertility. In addition, these activities can be comfortably added to that list of lifestyle recommendations and "things to avoid" as men attempt to conceive.

We believe that the finding of significantly improved semen quality in almost half of study patients is certainly evidence of an exposure effect that is more than just "occasional," as suggested above. And let us examine the clinical impact of this finding bit closer. How could the ability of a simple lifestyle maneuver that can "shift the care" from higher (IVF-ICSI) to lower and less expensive forms of assisted reproduction (IUI) not have the potential for enormous clinical impact given the cost of treating human subfertility? Just ask patients!

What we also find provocative is the discussion of possible clinical features that might impair the response to wet heat elimination (i.e. varicocele and tobacco use). Given the complexity of factors underlying male infertility, including currently uncharacterized issues, we agree that stronger, more generalizable statements regarding this particular exposure and its effect on male infertility can only be made with a larger study. 